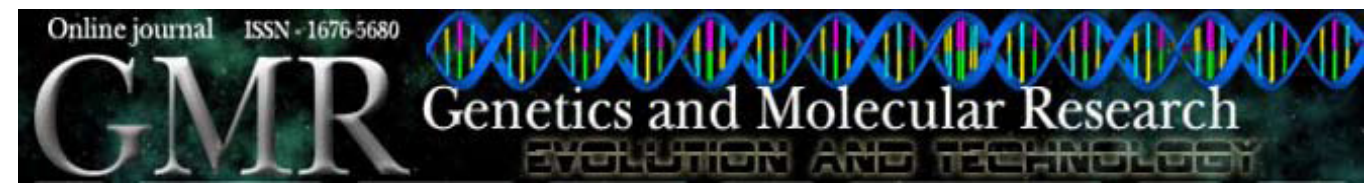

$\underline{\text { Review article }}$

\title{
Genetically modified animals for use in research and biotechnology
}

\author{
L.M. Chaible ${ }^{1}$, M.A. Corat ${ }^{2}$, E. Abdelhay ${ }^{3}$ and M.L.Z. Dagli ${ }^{1}$ \\ ${ }^{1}$ Laboratório de Oncologia Experimental, \\ Faculdade de Medicina Veterinária e Zootecnia, \\ Universidade de São Paulo, São Paulo, SP, Brasil \\ ${ }^{2}$ Centro Multidisciplinar para Investigação Biológica, \\ Universidade Estadual de Campinas, Campinas, SP, Brasil \\ ${ }^{3}$ Centro de Transplantes de Medula Óssea, \\ Instituto Nacional do Câncer, Rio de Janeiro, RJ, Brasil \\ Corresponding author: M.L.Z. Dagli \\ E-mail:mlzdagli@usp.br
}

Genet. Mol. Res. 9 (3): 1469-1482 (2010)

Received April 21, 2010

Accepted May 21, 2010

Published July 27, 2010

DOI 10.4238/vol9-3gmr867

\begin{abstract}
Transgenic animals are used extensively in the study of in vivo gene function, as models for human diseases and in the production of biopharmaceuticals. The technology behind obtaining these animals involves molecular biology techniques, cell culture and embryo manipulation; the mouse is the species most widely used as an experimental model. In scientific research, diverse models are available as tools for the elucidation of gene function, such as transgenic animals, knockout and conditional knockout animals, knock-in animals, humanized animals, and knockdown animals. We examined the evolution of the science for the development of these animals, as well as the techniques currently used in obtaining these animal models. We review the phenotypic techniques used for elucidation of alterations caused by genetic modification. We also investigated the role of genetically modified animals in the biotechnology industry, where they promise a revolution in obtaining heterologous proteins through natural secretions, such as milk, increas-
\end{abstract}


ing the scale of production and facilitating purification, thereby lowering the cost of production of hormones, growth factors and enzymes.

Key words: Transgenic animals; Knockout animals; Biotechnology; Disease models

\section{INTRODUCTION}

Science has advanced through observation and experimentation. So has the evolution of biotechnology. The emergence of animal models through genetic manipulation has greatly helped the development of scientific and biomedical knowledge. To speak of genetically modified animals is to focus on a very important chapter in the history of the science of laboratory animals, in which the function of genes was first identified in living individuals. Based on this knowledge, new ideas for treatment of genetic diseases and other diseases have emerged; these tend to revolutionize classic medicine, bringing benefits not only to man but to all living things.

Here we give a brief history of biotechnology and the production of genetically modified animals, as well as the types of animals that can be created, and findings derived from recombinant DNA technology and gene targeting.

\section{SOME HISTORICAL ASPECTS}

After 150 years of research, science has progressed from uncovering the principles of heredity to mimicking biological events in the laboratory. This information has provided significant advances in medicine over the last century, creating a new branch of industry, the billion-dollar biotechnology industry.

Since the initial genetic studies of Gregor Mendel in 1865, numerous researchers have contributed to the understanding and consolidation of his idea, which until then was only promising. In these decades of studies, important events took place, such as the discovery of the relationship of DNA with the transmission of genetic traits, the characterization of its chemical structure and of its three-dimensional structure, methodology to produce recombinant DNA, DNA cloning and sequencing, new animal models with random and, later, directed mutations, allowing the production of the first transgenic mice.

\section{DEVELOPMENT OF GENETICALLY MODIFIED ANIMAL MODELS}

At the beginning of the 20th century, with the appearance of the first genetic studies involving animal models, it was found that interference in the exchange of genetic information between different animals of the same species is important. Phenotypic characteristics that were found in these mating programs resulted in hypotheses of inheritable mechanisms that could behave differently over the generations, depending on the dominance and penetrance of these characteristics in individuals.

These observations showed the importance of the animal model in phenotype studies and encouraged the creation of new models that could respond to various biological questions. Through planned mating, diverse models appeared, including isogenic blood-relative models that could be used in a multitude of studies with little variability in the results. Over time, mu- 
tant animals appeared spontaneously in production colonies, differing from their parents and able to pass on these differences to their offspring. Other strains appeared from these spontaneous mutations and other models arose.

The possibility of enrichment of the animal models due to genetic alterations was evident. Contemporary researchers in the mid-20th century pursued various approaches to increase the possibility of genetic alterations in existing animal models and thus create new models. Chemical agents, such as base analogues, intercalating agents and agents reactive to DNA (ethyl methanesulfonate, methyl methane sulfate, methyl N-nitrosourea, and ethyl $\mathrm{N}$-nitrosourea) and also physical agents, such as radioactivity, were and still are widely used to produce forced random mutations in the animal genomes. Animals with phenotypic differences were then separated and their genomes completely analyzed to determine the genetic modifications involved with the phenotypic changes that were observed.

Taking a parallel path, Rudolf Jaenisch (1976) published the first report of introduction of an exogenous DNA virus (SV40) into mouse embryos, opening the door to the possibility of performing genetic alterations that were no longer random, but directed with the use of specific, chosen gene alterations.

Gordon and Ruddle (1981) reported the birth of a mouse that had been genetically modified through microinjection of purified DNA into single-cell mouse embryos. This methodology marked the birth of transgenesis and controlled genetic alteration in animal models. Thereafter, the growth in the production of genetically modified animal models by transgenesis has not stopped, and consequently the systemic knowledge of organisms and diseases has progressed with a speed unparalleled in history.

\section{TECHNIQUES FOR THE MANAGED CREATION OF GENETICALLY MODIFIED ANIMAL MODELS}

To create embryos in which the genome is permanently altered and the transfected DNA is permanently and irreversibly incorporated into the genetic material of the host cells, there are at least three important steps to be surmounted: 1) to produce a gene construct in vitro that is functional in accordance with the hypothesis of the proposed study; 2) to cause the required DNA to cross the plasma membrane, the cytoplasm and reach the nucleus, and 3) to cause the exogenous DNA to be integrated into the genome of the host cell and to be passed on unaltered to the following generations.

\section{Gene construct}

A gene construct can vary in complexity according to the insertion of interest. The construct can be designed to be inserted randomly into the genome of the animal, which is called transgenesis by addition, or can be designed to be inserted into the genome at a specific targeted site, into the correct position of a determined chromosome, which is called transgenesis by homologous recombination. In both cases, the construct must be impeccable, with structures to control gene expression, such as: a promoter, a site of transcription initiation, a site of polyadenylation, and a site of transcription termination. That is, the information that is being inserted into the receptor genome has a beginning, a middle, and an end, thus avoiding problems of uncontrolled expression in 
the host cell. In order to accomplish the objectives of the proposed genetic modification studies, a large variety of machinery is available today to control the expression of transgenes in a ubiquitous or conditional manner.

\section{Insertion of the DNA of interest into cells}

A variety of strategies can be used to cause exogenous DNA to penetrate the host cell.

Calcium precipitation. This is the original method described by Graham and Van der Eb (1973), in which the DNA is precipitated with calcium salts. The precipitated material is incorporated into endosomes, released into the cytoplasm and subsequently enters the nucleus. This method is fairly simple but very inefficient, mainly because much of the DNA is degraded within the endosome; however, it does succeed in some types of cell cultures.

Electroporation. This technique, described by Neumann et al. (1982), revolutionized the methodology of transfection of nucleic acids into cells in culture. The cells are mixed with DNA in solution and subjected to a sudden, powerful electric current, which causes the pores in the plasma membrane to open, allowing entry of the DNA. This is probably the most efficient physical method and can be applied to most cell types, it is also widely used.

Lipid micelles. Also known as lipoplexes, this methodology is based on the formation of complexes involving cationic lipid molecules of DNA. In addition to facilitating the passage of the genetic material through the cell membrane, it protects against the action of nucleases. It is one of the most popular and simple methods, requires no special equipment, is applicable to most cell types, but it is less efficient than electroporation.

Microinjection. This is the most widely used method in transgenesis for gene addition. In this method, the DNA in solution is physically injected into the cell nucleus. This method is considered $100 \%$ efficient to carry the DNA into the nucleus of somatic cells, but it has some disadvantages; it requires specialized equipment and highly skilled individuals, trained to perform the microinjection (Capecchi, 1980). This technique is widely used for insertion of gene vectors into fertilized oocytes, and it has a success rate of $4-8 \%$ of animals born with the transgene integrated into the genome (Brinster et al., 1985).

Viral vectors. This is the most recently employed method; it consists of the integration of the DNA of interest into the genome of a virus that is used to infect the cell. Among the viruses used are Simian virus 40 (SV40), retroviruses and lentiviruses. This method gives high rates of efficiency of expression (Hofmann et al., 2003).

\section{Integration}

Integration of exogenic DNA into the host genome occurs with extremely low efficiency, except in cases where the DNA to be transferred is contained in the viral particle (Hofmann et al., 2003). It is believed that the integration of exogenic DNA into the genome of the host cell occurs as a consequence of errors made when the DNA repair machinery of the host cell accidentally incorporates the exogenous molecule in an attempt to repair breaks in the double-stranded DNA that occasionally occur in cells (Brinster et al., 1985).

Without the use of viruses, the integration event is so rare that in cells that are success- 
fully transfected only a single event takes place; thus, the expression levels that are obtained are mostly a consequence of the locus of integration than directly to the number of copies inserted (Henikoff, 1998).

Due to the random nature of DNA breakage, integration of transfected DNA typically also occurs in random locations in the genome. Incorporated exogenous DNA could potentially disrupt, prevent or impede the expression of a gene of the host, although this probability is also rare. More frequently, the DNA integrates into a transcriptionally inactive region of the genome. Therefore, incorporation of DNA into the genome does not guarantee its expression. Even when expression occurs, it can often be unstable, gradually decrease over time, or sometimes express itself, or not, in an unforeseen way (Wilkie et al., 1986).

Due to these phenomena, it appears that only a fraction of the cells that integrate the exogenous DNA express the transgene, resulting in a mosaic pattern of expression. Positive selection techniques are used for the identification of cells positive for integration.

The predominant method of positive selection is through association of the gene of interest with a selection gene, for example, one that protects the cell from a toxic agent. The agent most often used for this purpose is a derivative of neomycin, called G418, which is added to the cell culture medium and inhibits cell growth. The cells that grow have the resistance gene NeoR integrated into the genome, which was inserted into the vector along with the transgene (Santerre et al., 1984).

\section{VECTOR CREATION}

Called a transgenesis vector, this structure is composed of nucleic acid, usually DNA, which is manipulated using molecular biology techniques. Vectors are derived from viruses and prokaryotic or eukaryotic plasmids that have the function of carrying the gene of interest into the host cell nucleus and integrating it into the genome.

Due to the complexity of gene regulation in animals, the best option is the use of the coding sequence of the gene, or cDNA containing the start codon (ATG), the complement of the mRNA of interest, thereby avoiding the requirement for post-transcription processing, splicing. For this coding sequence of the protein of interest to be efficiently expressed in mammalian cells, the following should be combined in the construct:

i) Promoter + upstream regulatory sequences - the combination of both is usually called "promoter". This region will determine the conditions of exogenous gene expression, as well as its intensity and location. Promoters may also be inducible, their activation mediated by drugs or by the physiological state of the animal.

ii) Coding sequence, which contains the genetic information for the messenger RNA (mRNA) to be formed. It is essential that there is an initiation codon (ATG) and a stop codon (TAA, TGA, TAG) in this sequence, which will signal the codons to be read in translation of mRNA by the ribosomal RNA (rRNA), resulting in the amino acid chain of the protein.

iii) Termination/polyA signaling sequence. The stop codon does not result in amino acids in the translation, acting as the stop signal of the process; the sequences TAG, TAA or TGA are used for this. The polyadenylation sequence triggers the addition of a repetition of adenosine at the end of the mRNA, providing stability to the molecule and allowing its translation. 


\section{TYPES OF GENETICALLY MODIFIED ANIMALS AND HOW THEY ARE PRODUCED}

\section{Transgenic animals}

Transgenic animals are those in which an exogenous gene was artificially inserted and stably incorporated into the genome of every cell of the organism and that can be transmitted to their descendants. The first transgenic mouse had the genome sequence of the Maloney leukemia virus inserted into its genome (Jaenisch, 1976).

The best way to get all the cells that compose the organism to express the exogenous gene is to insert it into cells that give rise to other cell types, i.e., the fertilized egg cell. In simple terms, the creation of transgenic animals begins with proper construction of the gene of interest and its transfection to the fertilized egg cell.

Many animal species have been genetically modified, including fish (Ozato et al., 1986), frogs (Ishibashi et al., 2008), rats (Agca et al., 2008), mice (Gordon and Ruddle, 1981; Joyner and Sedivy, 2000), cattle (Bondioli et al., 1991), birds (Etches and Verrinder Gibbins, 1997), and pigs (Kragh et al., 2009). The protocols to obtain such animals are diverse, but all consist of random integration of the exogenous gene into the genome of the animal. In most mammals, the exogenous DNA is inserted by pronuclear microinjection.

In mice, the most commonly used model, the frequency with which the exogenous DNA is incorporated into the genome is satisfactory in the case of the egg cell, a totipotent cell with a high capacity for cell division. Certain strains possess egg cells with larger pronuclei, providing a greater ease of microinjection. An example is the use of oocytes derived from FVB animals.

The animal generated from this egg cell, known as a transgenic founder, is heterozygous for the transgene, i.e., one of the chromosomes has incorporated the transgenic DNA, but not the homologous chromosome. Consequently, only half of the animals obtained from the F1 mating will also be heterozygous.

Transgenic models have diverse functions in research, in which the inserted gene may have its effects evaluated in vivo during the developmental stages, as in the evolution of diseases, in studies of mutations, in the search for therapies, and other parameters that necessitate an animal model for study. Industrially, the transgenic model can be used to obtain commercial productivity characteristics, and disease resistance, and can be used as a bioreactor in the production of biopharmaceuticals.

\section{Knockout animals}

The great contribution of these models was in the study of gene function through the phenotype presented due to the effect of allelic deletion. Until very recently, the only way to do this was to look for animals or humans suffering from hereditary diseases. In the case of animals, the incidence of such diseases could be increased by using mutagenic chemicals or irradiation, then the location of the mutation was mapped and the defective gene cloned.

Unlike the transgenic model produced by the addition of random exogenous DNA, the knockout model is obtained by targeted insertion. This targeted integration is performed through the mechanism of homologous recombination and has an extremely low success rate (Gama Sosa et al., 2010). 
Homologous recombination is a separate chapter in the methodology for obtaining knockout animals. This technique allows the insertion of a determined transgene into a specific locus, causing it to locate exactly in the desired portion of the chromosome. The most common way to delete exons is through the creation of a targeted construct, also called "targeted". In this case, the plasmid must contain a homologous portion of the start of the gene, known as "left arm" and a portion obtained along the gene, or even the final portion, called the "right arm" (Capechi, 1980).

Between these portions, an exon must be situated that is important for the functionality of the protein. These homologous regions will pair with the corresponding sequences in the gene of interest. The longer the arms, the greater the probability that the pairing will occur. In practice, the combined length of both arms is between 6 and $10 \mathrm{~kb}$ (Joyner and Sedivy, 2000). As a result of this pairing and a double recombination, one in each arm, the original gene, or "wild type" is replaced by the transgene contained between the two arms of the construct. The gene sequence to be introduced may contain another gene, usually a reference sequence of a positive selection gene, thus generating substitution of expression of the original gene for NeoR.

Due to the low efficiency of the occurrence of two recombinations so close together, the strategy of performing this process in cell culture is used; but for these cells to give rise in the future to a complete organism they need to be pluripotent cells. The ideal cells are known as ES cells (embryonic stem) (Smithies et al., 1985; Notarianni and Evans, 2006). It was found that these cells called "germ lines" were perfectly suited for targeted insertion and could be identified through mechanisms of positive and negative selection. Currently, protocols for the cultivation and maintenance of these cells exist for only a few species (Grivennikov, 2008).

ES cells can be transferred by micromanipulation into the interior of murine embryos at about three to four days of age, during the blastocyst pre-implantation phase. These modified cells will attach to the normal cells of the inner cell mass of the blastocyst and will behave as cells of the embryo itself, participating in the formation of the three germ layers of the individual (ectoderm, mesoderm and endoderm) and contributing to the formation of all tissues of the adult mouse. This creates what is known as a chimeric mouse, in which each organ is formed from both cells of the embryo itself and from the ES-injected cells, which will include the gonads, giving rise to germ line animals (Gama Sosa et al., 2010).

At present, strains of knockout mice for $10 \%$ of the genes have been produced, and ambitious projects like the KOMP (Knock-Out Mouse Project) and the EUCOMM (European Conditional Mouse Mutagenesis) aim to obtain strains with individual deletions for all genes, as well as mutations known to be related to syndromes.

\section{Conditional knockout animals}

Deletion of genes essential for development can result in embryonic lethality, due to lack of influence of this gene in the embryogenesis of the animal; thus, mice die in the uterus, making it difficult to study events that occur after birth, and impossible to study the function of these genes in adult animals. Fortunately there are strategies, which are now becoming prevalent, to circumvent this problem, aiming to avoid problems with embryonic lethality (Gama Sosa et al., 2010).

One possible strategy is the use of the conditional deletion system, which involves gene deletion under specific conditions. The main strategy is known as the Cre-Lox system. 
The Cre enzyme is a $38-\mathrm{kDa}$ recombinase, present in the $\mathrm{P} 1$ bacteriophage, which mediates recombination of DNA regions flanked by sequences such as loxP (Hamilton and Abremski, 1984). The loxP sequence has 34 bp with a defined orientation. When the loxP flanks are in consensus, the region contained between them is excised, leaving a single loxP sequence. When the flanking sites are in opposite positions, the Cre enzyme recombines the contained region, reversing it. That is, animals with a targeted insertion in the same position as the loxP flanks are obtained by means of homologous recombination in ES cells. This insertion gene may be the same gene present in the locus, but now flanked by the loxP sequence. The situation that will permit its normally conditional expression is expression of Cre recombinase, which may be ubiquitous or conditioned to a determined organ or cell type, and eliminates the flanked gene. This Cre recombinase gene can be inherited from another transgenic animal and will allow conditional expression of this protein.

This conditional control is based on the Cre enzyme, which may have its expression controlled through the promoter related to it. The promoter that regulates the expression of the Cre gene will control the pattern of deletion of the target gene. This promoter may be specific to cell types, the physiological state of the animal or inducible by drugs.

i) Cell-specific promoter. The regulatory region of the Cre gene is important in determining the cell or group of cells that express the recombinase. Using the aP2 promoter, it is possible to delete the gene of interest only in adipocytes, the $\alpha \mathrm{MHC}$ promoter for deletion in cardiomyocytes, the ALB promoter for hepatic cells, the Nestin promoter for neurons, the K5 promoter for epithelial cells of the skin, the CD19 promoter for B lymphocytes, and the Cola1 promoter for osteoblasts.

ii) Temporal promoters. Regulatory sequences that promote gene expression at specific periods or physiological states. The CaMKII promoter can be used for gene deletion in neurons only after birth.

iii) Inducible promoters. Regulatory sequences that are activated or deactivated by exogenous molecules. The TET system of induction, based on the TET operon system of Escherichia coli, is the most widely used (Sun et al., 2007). With this strategy, the Cre enzyme can be expressed, and consequently delete the gene at a specific time according to the research objective. To accomplish this, tet-Cre animals are crossed with loxP animals, and after mating of homozygous flanked animals, tetracycline or its analogue, doxycycline, is administered, excising the gene of interest and obtaining the desired knockout at the targeted systemic site, at the determined time of development of the animal.

\section{Knock-in animals}

As described, these techniques are most frequently used to introduce genes into random locations (transgenics) or to delete specific portions of genes (targeted knockout). The same techniques can be used to introduce new genetic elements into specific sites, known as knock-in (Haruyama et al., 2009). One application of this technique is to study the expression of molecules, identifying cells that express and modulate expression, using reporter molecules for this. The most commonly used reporter is the enzyme $\beta$-galactosidase, which can be detected with a variety of colored substrates, and the "green fluorescent protein", also known as GFP (Welsh and Kay, 1997).

For this, after the stop codon sequence of the studied gene, an entry site within the ri- 
bosome is added, termed IRES (internal ribosome entry site), followed by the cDNA sequence of the reporter gene. The IRES sequence has the property of permitting a single molecule of eukaryotic mRNA to code for two different proteins.

Knock-in animals are also fundamental models in other studies. Through targeted insertion the wild sequence of a targeted gene can be modified to a mutated sequence, related to disorders and other syndromes (Davis et al., 2007; Desbonnet et al., 2009).

A very interesting technical use of knock-in is the insertion of a target gene into the locus of another gene, substituting it. Some genes belong to a large family, but the members display individual characteristics, which often preclude compensation. Zheng-Fischhöfer et al. (2006), in an attempt to avoid embryonic lethality in knockout animals for the $\mathrm{Cx} 43$ gene, substituted the loci deleted with the $\mathrm{Cx} 31$ gene, producing the knock-in animal $\mathrm{Cx} 43^{31 / 31}$. The authors perceived that despite the relative gene homology, the restoration of cell communication via $\mathrm{Cx} 31$ was not sufficient for normal development of the heart, showing selectivity of size and electrical charge between proteins $\mathrm{Cx} 43$ and $\mathrm{Cx} 31$.

\section{Mice that host fragments of chromosomes}

Technologies developed in recent decades have made possible the manipulation of artificial chromosomes such as PACs, YACs and BACs, allowing the use of sequences with hundreds of kilobases originating from human genomes, those of mice and many other species for animal transgenesis (Sparwasser and Eberl, 2007).

The first mouse genetically modified by BACs was obtained by Yang et al. (1997); it allowed mapping the expression of various genes through co-expression of reporter genes. Later studies optimized the technique by integrating it with other methods of transgenesis.

During the 1990s, many experiments were conducted using artificial chromosomes, in order to obtain mice genetically modified with human genes (Sparwasser and Eberl, 2007). This humanized mouse is useful for the study of the expression of factors related to the immune system, identification of genes related to human diseases and the creation of new disease models (Kazuki et al., 2003; Li et al., 2009).

One important use of these animals would be the ability to produce human antibodies that could be injected into patients without the possibility of being recognized, or even destroyed, by the immune system of the individual. Such antibodies could be used to rapidly provide protection against dangerous pathogens, or be directed against critical surface molecules of $\mathrm{T}$ cells to act as immunosuppressive agents during organ transplants.

\section{Knockdown mice using RNA interference technology}

The knockdown of genes, either in a constitutive or a conditional manner, is performed using RNA interference (RNAi) techniques. The first steps for understanding the RNAi technique came from data from invertebrate studies. Long molecules of double-stranded RNA (dsRNA) were seen to be processed into small interfering RNAs (siRNAs) by the ribonuclease, Dicer. The antisense siRNA served as a standard for the complex called RISC (RNAinduced silencing complex), which recognizes and cleaves the complementary mRNA, which consequently is rapidly degraded (Fire et al., 1998). In mammals, dsRNAs longer than 30 bp provoke a global response of inhibition of protein synthesis and non-specific degradation 
of mRNA. However, small synthetic molecules of dsRNAs can serve as triggers for specific breaks of mRNAs in animal cells.

The strategy for production of a knockdown animal for a particular gene begins with the design of the vector. The vector must contain a sequence that encodes an RNA transcript complementary to the target mRNA and must contain a hairpin region, forming an shRNA, a key feature for processing by the Dicer enzyme and subsequent formation of siRNA (Hitz et al., 2009). The vector should be integrated into the genome so that the knockdown is permanent and is transmitted through generations.

The big difference between the knockdown and the knockout model is the type of deletion caused. Knockout animals have the sequence responsible for mRNA production deleted from the genome, while in the knockdown animals, the interference process acts during the post-transcriptional phase. This characteristic ensures that the target protein is completely absent in the knockout animals, while in the knockdown model that efficiency is lower, achieving up to a $90 \%$ decrease in protein quantity (Hitz et al., 2009).

In some cases, permanent knockdown of the target protein is not required because of malformation of the animal or even for the understanding of gene function. In such cases, we can associate the expression of RNAi with an inducible promoter controlled by doxycycline (Tet-On inducible expression system), thus obtaining the knockdown for specific periods (Dickins et al., 2007).

\section{USE OF GENETICALLY MODIFIED ANIMALS IN SCIENTIFIC RESEARCH}

At the beginning of the last century, the science of genetics was still in formation, but animals were already domesticated, bred and genetically selected in animal facilities for use in research on anatomy, physiology, reproduction, and immunology. Recently, humanity has gone through an era of controversy, because, at the same time that scientists say that it is essential to use live animals for experimentation, diverse groups say they are opposed to their use. The fact is that in vitro models equivalent to animal models currently do not exist. The complexity of a living creature cannot be mimicked in vitro, and therefore there is a consensus among scientists that the use of these animals is ethical. Among the animal species, the mouse is considered to be the best model for studying human diseases and therefore the one most employed. This choice is based on characteristics such as: small size, short life cycle, the possibility of genomic manipulation, many molecular and physiological similarities with humans, human orthologues mapped, and the complete genome sequenced (Rosenthal and Brown, 2007).

Until the 1970s, the only way to study genetic diseases was with the identification of animals with spontaneous mutations that showed phenotypic changes similar to a disease in humans. With the advance of genetic engineering, it was no longer necessary to wait; these animals could be produced with the desired alterations, since the genetic causes of the disease in question were known. So, animals were produced that serve as models for studies of different types of cancers (Santos et al., 2008), heart disease (Moon, 2008), hypertension (Zadelaar et al., 2007), diabetes (Leroith and Gavrilova, 2006), obesity (Blüher, 2005), osteoporosis (Klein, 2008), glaucoma (Zhou et al., 2008), blindness (Moussaif et al., 2006), deafness (Leibovici et al., 2008), Huntington's syndrome (Heng et al., 2008), Down's syndrome (Patterson, 2009), Parkinson's disease (Harvey et al., 2008), Alzheimer's disease (Gotz et al., 2009), anxiety (Kalueff et al., 2007), and depression (Kalueff et al., 2007). 
After deciphering the sequence of nucleotides that compose the genomes of humans, mice and other species, the challenge for researchers is understanding the genes and their functions, interactions, expression patterns, and their correlation with diseases and syndromes. Without doubt, the models discussed here will be vital in these processes, i.e., knock-in, constitutive knockout, conditional knockout, or knockdown animals.

After the creation of the model, the next step is a more complex, time-consuming and practically unlimited process, phenotyping. Phenotyping can be divided into three steps:

1 - Clinical and morphological characterization. Evaluation of all parameters related to normal functioning of the organism as well as morphological characteristics, such as: heart rate, respiratory rate, blood pressure, behavioral analysis, histological and organ analysis, auditory and ophthalmic analysis, monitoring of growth and development of the animal, profile of serum molecules, immune and reproductive systems.

2 - Molecular characterization. This process will principally evaluate three types of molecules: DNA, mRNA and proteins, all related to the mechanism of transgenesis. In some models, it is essential to identify the region of integration of the vector into the genome of the animal, as well as the number of copies inserted, which can be accomplished using in situ hybridization. The qPCR technique is used to evaluate the pattern and level of expression of mRNA in cells. Western blot, ELISA or radioimmunoassay techniques are used to evaluate the levels of proteins produced, while immunohistochemistry is employed to analyze the location and proper functionality of the proteins.

3 - Pathological characterization. This step will study the possibilities of participation of the altered gene in the spontaneous onset of disease or ease of onset during their induction. Examples of diseases include cancer, diabetes, hypertension, cardiac problems, renal problems, anemia, autoimmune diseases, and allergies.

\section{USE OF GENETICALLY MODIFIED ANIMALS IN THE PRODUCTION OF BIOPHARMACEUTICALS}

The production of high-value pharmaceutical substances, including recombinant proteins, has a value of billions of dollars per year around the world; in this context, genetically modified animals promise to revolutionize the pharmaceutical industry. The heterologous proteins produced in bioreactors are normally hormones, growth factors and enzyme complexes. Transgenic animals are indicated as potential bioreactors for the synthesis of heterologous protein complexes. They are capable of post-translational modifications, such as glycosylations, in a similar way to what occurs naturally in humans, more reliably than other systems, such as yeast. Many fluids have been tested, such as blood, urine, seminal plasma, egg white, and milk.

The mammary gland can be considered the most natural and efficient producer of heterologous proteins. Many studies have demonstrated that recombinant proteins can be produced in milk, including human IGF-1 (Zinovieva et al., 1998), hGH (Devinoy et al., 1994), human lysozyme (Lee et al., 1998), human lactoferrin (Platenburg et al., 1994), human erythropoietin (Sohn et al., 1999), human parathyroid hormone (Rokkones et al., 1995), and coagulation factor VIII (Chrenek et al., 2007).

Mammals that serve as bioreactors are genetically modified to produce the heterologous protein exclusively in the mammary gland, optimizing its production and avoiding deleterious effects of the biopharmaceuticals on the health of these animals. Through selective 
promoters for the mammary gland, such as as 1-casein (Platenburg et al., 1994; Zinovieva et al., 1998) and whey acid protein - WAP (Devinoy et al., 1994; Rokkones et al., 1995), gene expression can be targeted to the mammary gland, with the release of the desired protein in the milk.

The purification of recombinant protein in milk is generally not a problem. Milk does not contain large amounts of proteases and the casein can be removed easily, without aggressive procedures. A chromatograph can, in most cases, eliminate most unwanted proteins, leaving the target protein with a high level of purity. However, it should be considered that milk is a biological complex with many components, and complete purification in some cases can be very difficult. The biosecurity of the biopharmaceuticals thus obtained can be assured by means of sanitary control of livestock, especially animals free of contamination with prions.

Many animal species have been used as bioreactors, including mice, cattle, pigs, goats, sheep, and rabbits. The transgenesis of production animals is still in development; some important steps have been taken, but this still requires the development of stronger promoters to ensure adequate efficiency, thus reducing transgenesis costs and enabling this procedure to be carried out on an industrial scale. With the new technologies of manipulation of bovine embryos, commercial cloning and the sequencing of the bovine genome, production of these genetically modified animals is the next step. In a few years, genetic improvement of these animals will be attained at a much faster rate than is possible today, enabling the production of animal-origin foods with elevated nutritional properties, more resistance to decomposition and with better organoleptic characteristics.

\section{BIOSECURITY IN OBTAINING AND REARING GENETICALLY MODIFIED ANIMALS}

According to Brazilian legislation concerning genetically modified organisms, the Biosecurity Law (law 11105 of March 24, 2005 and Decree No. 5.591 of November 22, 2005), to develop, create or use a genetically modified animal, the animal facility and laboratory must have a Certificate of Quality in Biosecurity, or CQB, which is awarded by the National Technical Commission on Biosecurity (CTNBio) of the Ministry of Science and Technology. The institution must also appoint an Internal Biosecurity Commission (CIBio) to manage activities involving genetically modified organisms. The laboratories should be equipped for biosecurity at a level equivalent to the risk class of the genes or agents with which it will work, and the staff must be trained and educated to adequately carry out genetic modifications. The disposal of materials is extremely important; this material must be inactivated prior to disposal in the environment.

Various forms of inactivation of genes and/or vectors can be found, but the autoclave is one of the most secure and widely used. The animal facility should also possess some specific features to accommodate the genetically modified animals. The animals must be kept in separate, locked rooms, the doors of which must bear the biosecurity symbol and information on which animals are kept there. Animals should be housed in cages for easy cleaning and decontamination, which should be well sealed to prevent the escape of animals or the entry of other animals into the cage. Ventilated shelving or racks, or microisolators, are suitable for the maintenance of genetically modified animals. After euthanasia, the corpses of genetically modified animals should be sent for incineration along with medical waste. All accidents (e.g., escape of animals, spill of liquids originating from genetically modified animals) must be reported to the CIBio of the unit, which in turn should report them to CTNBio. 
Most genetically modified animals belong to risk class 1 and thus can be produced and reared in laboratories or animal facilities that are biosecurity level 1. It is always important to remember that all rules of bioethics applicable to conventional laboratory animals (such as methods of euthanasia and attitudes that prevent the suffering of animals) should also be applied to genetically modified animals.

\section{REFERENCES}

Agca C, Fritz JJ, Walker LC, Levey AI, et al. (2008). Development of transgenic rats producing human beta-amyloid precursor protein as a model for Alzheimer's disease: transgene and endogenous APP genes are regulated tissuespecifically. BMC Neurosci. 9: 28.

Blüher M (2005). Transgenic animal models for the study of adipose tissue biology. Best Pract. Res. Clin. Endocrinol. Metab. 19: 605-623.

Bondioli KR, Biery KA, Hill KG, Jones KB, et al. (1991). Production of transgenic cattle by pronuclear injection. Biotechnology 16: 265-273.

Brinster RL, Chen HY, Trumbauer ME, Yagle MK, et al. (1985). Factors affecting the efficiency of introducing foreign DNA into mice by microinjecting eggs. Proc. Natl. Acad. Sci. U.S.A. 82: 4438-4442.

Capecchi MR (1980). High efficiency transformation by direct microinjection of DNA into cultured mammalian cells. Cell 22: 479-488.

Chrenek P, Ryban L, Vetr H, Makarevich AV, et al. (2007). Expression of recombinant human factor VIII in milk of several generations of transgenic rabbits. Transgenic Res. 16: 353-361.

Davis RE, Swiderski RE, Rahmouni K, Nishimura DY, et al. (2007). A knockin mouse model of the Bardet-Biedl syndrome 1 M390R mutation has cilia defects, ventriculomegaly, retinopathy, and obesity. Proc. Natl. Acad. Sci. U.S.A. 104: 19422-19427.

Desbonnet L, Waddington JL and O'Tuathaigh CM (2009). Mutant models for genes associated with schizophrenia. Biochem. Soc. Trans. 37: 308-312.

Devinoy E, Thepot D, Stinnakre MG, Fontaine ML, et al. (1994). High level production of human growth hormone in the milk of transgenic mice: the upstream region of the rabbit whey acidic protein (WAP) gene targets transgene expression to the mammary gland. Transgenic Res. 3: 79-89.

Dickins RA, McJunkin K, Hernando E, Premsrirut PK, et al. (2007). Tissue-specific and reversible RNA interference in transgenic mice. Nat. Genet. 39: 914-921.

Etches RJ and Verrinder Gibbins AM (1997). Strategies for the production of transgenic chicken. Methods Mol. Biol. 62: 433-450.

Fire A, Xu S, Montgomery MK, Kostas SA, et al. (1998). Potent and specific genetic interference by double-stranded RNA in Caenorhabditis elegans. Nature 391: 806-811.

Gama Sosa MA, De Gasperi R and Elder GA (2010). Animal transgenesis: an overview. Brain Struct. Funct. 214: 91-109.

Gordon JW and Ruddle FH (1981). Integration and stable germ line transmission of genes injected into mouse pronuclei. Science 214: 1244-1246.

Gotz J, Schonrock N, Vissel B and Ittner LM (2009). Alzheimer's disease selective vulnerability and modeling in transgenic mice. J. Alzheimers Dis. 18: 243-251.

Graham FL and van der Eb AJ (1973). Transformation of rat cells by DNA of human adenovirus 5. Virology 54: 536-539.

Grivennikov IA (2008). Embryonic stem cells and the problem of directed differentiation. Biochemistry 73: 1438-1452.

Hamilton DL and Abremski K (1984). Site-specific recombination by the bacteriophage P1 lox-Cre system. Cre-mediated synapsis of two lox sites. J. Mol. Biol. 178: 481-486.

Haruyama N, Cho A and Kulkarni AB (2009). Overview: engineering transgenic constructs and mice. Curr. Protoc. Cell Biol. (Chapter 19): Unit.

Harvey BK, Wang Y and Hoffer BJ (2008). Transgenic rodent models of Parkinson's disease. Acta Neurochir. Suppl. 101: 89-92.

Heng MY, Detloff PJ and Albin RL (2008). Rodent genetic models of Huntington disease. Neurobiol. Dis. 32: 1-9.

Henikoff S (1998). Conspiracy of silence among repeated transgenes. Bioessays 20: 532-535.

Hitz C, Steuber-Buchberger P, Delic S, Wurst W, et al. (2009). Generation of shRNA transgenic mice. Methods Mol. Biol. 530: 101-129.

Hofmann A, Kessler B, Ewerling S, Weppert M, et al. (2003). Efficient transgenesis in farm animals by lentiviral vectors. EMBO Rep. 4: 1054-1060. 
Ishibashi S, Kroll KL and Amaya E (2008). A method for generating transgenic frog embryos. Methods Mol. Biol. 461: 447-466. Jaenisch R (1976). Germ line integration and Mendelian transmission of the exogenous Moloney leukemia virus. Proc. Natl. Acad. Sci. U.S.A. 73: 1260-1264.

Joyner AL and Sedivy JM (2000). Gene Targeting: A Practical Approach. Oxford University Press, Oxford.

Kalueff AV, Wheaton M and Murphy DL (2007). What's wrong with my mouse model? Advances and strategies in animal modeling of anxiety and depression. Behav. Brain Res. 179: 1-18.

Kazuki Y, Schulz TC, Shinohara T, Kadota M, et al. (2003). A new mouse model for Down syndrome. J. Neural Transm. Suppl. 67: 1-20.

Klein RF (2008). Genetics of osteoporosis - utility of mouse models. J. Musculoskelet. Neuronal Interact. 8: 287-290.

Kragh PM, Nielsen AL, Li J, Du Y, et al. (2009). Hemizygous minipigs produced by random gene insertion and handmade cloning express the Alzheimer's disease-causing dominant mutation APPsw. Transgenic Res. 18: 545-558.

Lee WK, Kim SJ, Lee T and Han Y (1998). Expression of a bovine beta-casein/human lysozyme fusion gene in the mammary gland of transgenic mice. J. Biochem. Mol. Biol. 31: 413-417.

Leibovici M, Safieddine S and Petit C (2008). Mouse models for human hereditary deafness. Curr. Top. Dev. Biol. 84: 385-429.

Leroith D and Gavrilova O (2006). Mouse models created to study the pathophysiology of type 2 diabetes. Int. J. Biochem. Cell Biol. 38: 904-912.

Li Y, Liu W, Oo TF, Wang L, et al. (2009). Mutant LRRK2(R1441G) BAC transgenic mice recapitulate cardinal features of Parkinson's disease. Nat. Neurosci. 12: 826-828.

Moon A (2008). Mouse models of congenital cardiovascular disease. Curr. Top. Dev. Biol. 84: 171-248.

Moussaif M, Rubin WW, Kerov V, Reh R, et al. (2006). Phototransduction in a transgenic mouse model of Nougaret night blindness. J. Neurosci. 26: 6863-6872.

Neumann E, Schaefer-Ridder M, Wang Y and Hofschneider PH (1982). Gene transfer into mouse lyoma cells by electroporation in high electric fields. EMBO J. 1: 841-845.

Notarianni E and Evans MJ (2006). Embryonic Stem Cells: A Practical Approach. Oxford University Press, Oxford.

Ozato K, Kondoh H, Inohara H, Iwamatsu T, et al. (1986). Production of transgenic fish: introduction and expression of chicken delta-crystallin gene in medaka embryos. Cell Differ. 19: 237-244.

Patterson D (2009). Molecular genetic analysis of Down syndrome. Hum. Genet. 126: 195-214.

Platenburg GJ, Kootwijk EP, Kooiman PM, Woloshuk SL, et al. (1994). Expression of human lactoferrin in milk of transgenic mice. Transgenic Res. 3: 99-108.

Rokkones E, Fromm SH, Kareem BN, Klungland H, et al. (1995). Human parathyroid hormone as a secretory peptide in milk of transgenic mice. J. Cell Biochem. 59: 168-176.

Rosenthal N and Brown S (2007). The mouse ascending: perspectives for human-disease models. Nat. Cell Biol. 9: 993-999.

Santerre RF, Allen NE, Hobbs JN Jr, Rao RN, et al. (1984). Expression of prokaryotic genes for hygromycin B and G418 resistance as dominant-selection markers in mouse L cells. Gene 30: 147-156.

Santos J, Fernandez-Navarro P, Villa-Morales M, Gonzalez-Sanchez L, et al. (2008). Genetically modified mouse models in cancer studies. Clin. Transl. Oncol. 10: 794-803.

Smithies O, Gregg RG, Boggs SS, Koralewski MA, et al. (1985). Insertion of DNA sequences into the human chromosomal beta-globin locus by homologous recombination. Nature 317: 230-234.

Sohn BH, Kim SJ, Park H, Park SK, et al. (1999). Expression and characterization of bioactive human thrombopoietin in the milk of transgenic mice. DNA Cell Biol. 18: 845-852.

Sparwasser T and Eberl G (2007). BAC to immunology - bacterial artificial chromosome-mediated transgenesis for targeting of immune cells. Immunology 121: 308-313.

Sun Y, Chen X and Xiao D (2007). Tetracycline-inducible expression systems: new strategies and practices in the transgenic mouse modeling. Acta Biochim. Biophys. Sin. 39: 235-246.

Welsh S and Kay SA (1997). Reporter gene expression for monitoring gene transfer. Curr. Opin. Biotechnol. 8: 617-622.

Wilkie TM, Brinster RL and Palmiter RD (1986). Germline and somatic mosaicism in transgenic mice. Dev. Biol. 118: 9-18.

Yang XW, Model P and Heintz N (1997). Homologous recombination based modification in Escherichia coli and germline transmission in transgenic mice of a bacterial artificial chromosome. Nat. Biotechnol. 15: 859-865.

Zadelaar S, Kleemann R, Verschuren L, de Vries-Van der Weij, et al. (2007). Mouse models for atherosclerosis and pharmaceutical modifiers. Arterioscler. Thromb. Vasc. Biol. 27: 1706-1721.

Zheng-Fischhöfer Q, Ghanem A, Kim JS, Kibschull M, et al. (2006). Connexin31 cannot functionally replace connexin43 during cardiac morphogenesis in mice. J. Cell Sci. 119: 693-701.

Zhou Y, Grinchuk O and Tomarev SI (2008). Transgenic mice expressing the Tyr437His mutant of human myocilin protein develop glaucoma. Invest. Ophthalmol. Vis. Sci. 49: 1932-1939.

Zinovieva N, Lassnig C, Schams D, Besenfelder U, et al. (1998). Stable production of human insulin-like growth factor 1 (IGF-1) in the milk of hemi- and homozygous transgenic rabbits over several generations. Transgenic Res. 7: 437-447. 